\title{
Development of a Path-loss Prediction Model Using Adaptive Neuro- fuzzy Inference System
}

\author{
Adeyemo Z.K ${ }^{\mathrm{a}}$, Olawuyi T.O ${ }^{\mathrm{b}}$, Oseni O.F ${ }^{\mathrm{c}}$, Ojo S.I ${ }^{\mathrm{d}}$ \\ ${ }_{a, b, c, d}$ Department of Electronic and Electrical Engineering Ladoke Akintola University of Technology, \\ Ogbomoso, Nigeria
}

Received: 20 August 2019; Accepted: 15 October 2019; Published: 08 November 2019

\begin{abstract}
The prediction of wireless communication signals is of paramount importance for proper network planning. The existing prediction models such as Okumura-Hata, Co-operative for Scientific and Technical Research (COST-231) and free space are less accurate for predicting path-loss values of wireless signals due to differences in propagation environments. Hence, this paper develops a path-loss model using Adaptive NeuroFuzzy Inference System (ANFIS) for accurate prediction of wireless High Speed Packet Access (HSPA) network signal in Ibadan, Nigeria. This is achieved by measuring the Received Signal Strength (RSS) from three Base Transmitting Stations (BTS) operating at $2100 \mathrm{MHz}$ frequency in Ojo (longitude E 3' 53.1060', latitude N 7'27.2558'), Dugbe (longitude E 3'50.4361', latitude N 7' 23.0678') and Challenge (longitude E 3' 53.1060', latitude N 7' 21.258') areas of Ibadan using the Drive Test. Ericson Test Equipment for Mobile System (TEMS) phone, Global Positioning System (GPS) and Computer System are used to obtain RSS data at different distances. Base station parameters such as the transmitting antenna height, receiving antenna height, carrier frequency and distance are used as input variables to train ANFIS to develop a model. These base station parameters are also used to investigate the suitability of Okumura-Hata, COST-231 and free space model. A five layer ANFIS structure is developed and trained using Least Square Error (LSE) and Gradient Descent (GD) method to adjust the consequent and premise parameters. The performance of the developed ANFIS model is evaluated using Mean Square Error (MSE) and Root Mean Square Error (RMSE) and compared with Okumura-Hata, COST 231 and free space. The results obtained for ANFIS give lower RMSE and MSE indicating the suitability of ANFIS model for path-loss prediction. The developed ANFIS model can be used for network planning and budgeting in these environments.
\end{abstract}

Index Terms: HSPA, ANFIS, Least Square Error, Gradient Descent method

(C) 2019 Published by MECS Publisher. Selection and/or peer review under responsibility of the Research Association of Modern Education and Computer Science

\footnotetext{
* Corresponding author. Tel: +23408033889921, +23407031819045

E-mail address: zkadeyemo@lautech.edu.ng, olawuyitolulope1513@gmail.com
} 


\section{Introduction}

Wireless communication is the transmission of signals over a wireless channel that has no physical connection between the transmitter and the receiver [1]. There is reduction in signal strength as the distance increases indicating the loss in signal along the path. To determine the actual path-loss, path-loss models have been developed for predicting the values. These models are mathematical expressions used to predict the radio signal characteristics of a place [2]. The models are categorized into three; random, deterministic and empirical models [1,7,9]. Random models generate predictions randomly without proper considerations of all the physical environmental phenomena which make it unreliable when applied to environment that has complex structure and many obstacles [9,12]. Deterministic models rely on ray optical laws for prediction and are computationally complex and time consuming $[11,12]$. Although these models seem to be accurate than other existing model but are difficult to implement especially in environments where there are many obstacles along the signal paths.

\begin{tabular}{|ll|}
\hline Nomenclature & \\
AI & Artificial Intelligence \\
ANFIS & Adaptive Neuro-Fuzzy Inference System \\
ANN & Artificial Neural Network \\
BTS & Base Transmitting Stations \\
COST & Co-operative for Scientific and Technical Research \\
FL & Fuzzy Logic \\
GD & Gradient Descent \\
GPS & Global Positioning System \\
HSPA & High Speed Packet Access \\
LSE & Least Square Error \\
MSE & Mean Square Error \\
RMSE & Root Mean Square Error \\
RSS & Received Signal Strength \\
SM & Stochastic Model \\
SUI & Stanford University Interim \\
\hline
\end{tabular}

On the other hand, Empirical models are dependent on the environment where the measurement is taken. To make these models applicable to other environments, optimization approach must be carried out on the models for proper adaption $[3,8,12,18,19]$. The measured data is used as reference for comparison with optimized models. A major limitation of the models is large deviation when compared to the measured data. Therefore, this paper investigates the applicability of the existing models in Ibadan, Nigeria and the development of a new model using Adaptive Neuro-Fuzzy Inference System (ANFIS). The investigation is carried out using the drive test method. Drive test equipment such as Test Equipment for Mobile System (TEMS), a computer system and a Global Positioning System (GPS) are used to carry out the measurement along three different locations in Ibadan, Nigeria. Path-loss values obtained from measured RSS are used to determine the deviation from pathloss values of the existing models. An ANFIS structure was developed and trained with transmitting antenna height, receiving antenna height, carrier frequency and distance as the input variables. Least Square Error (LSE) and Gradient Descent (GD) method are used to adjust the consequent and premise parameters. The new model developed using ANFIS is compared to the measured path-loss values. 


\section{Related works}

In [7], Fuzzy Logic (FL) approach was used for path-loss prediction in Mehuwala, India. A path-loss exponent was assigned to each propagation environment. The authors classified the propagation medium into several propagation environments. A path-loss exponent (n) was assigned to each propagation environment. The result was compared to Hata model using path-loss exponent. Path-loss increased at the rate of $26.7 \mathrm{~dB}$ per decade with distance for FL Model. The rate of increase of path-loss per decade with distance for Hata model is $114.8 \mathrm{~dB}$. The authors concluded that FL Approach is more efficient in path-loss prediction than theoretical analysis. In [10], a model was developed for Hyderabad city using Artificial Neural Network (ANN). The authors trained the measured data using Feed Forward Neural Network (FFNN). Leverberg Marquart algorithm was used for the training. The result was presented and compared to Hata, COST-231 and Walfisc model. ANN results were found to be better than other models.

In [16], FL was used to estimate path-loss in Dehradum and Uttarakh environments at India. The authors assigned a mean path-loss exponent (n) to each of the environment such as free space terrain, flat area terrain, heavy terrain and light terrain. Data was taken in three Zones within $1 \mathrm{~km}$ to $5 \mathrm{~km}$ Transmitter-Receiver separation distance. Path-loss exponent (n) was calculated by linear regression such that the error between measured and estimated path-loss is minimized. Finally, FL model was compared to Hata, COST-231 and Electronic Communication Committee (ECC-33) models using path-loss exponents (n). FL method proved a different method in path-loss prediction. The author concluded that, theoretical analysis is said to be different from practical analysis and to achieve a better result, the two must be combined. In [17], Adaptive NeuroFuzzy Inference System approach was used for path-loss prediction at VHF band. A five layer ANFIS structure was trained with frequency and distance as the input variables. Performance of the developed ANFIS model was compared to COST-231, Egli and ECC-33 models. ANFIS was found to have better predictions in terms of RMSE, Spread Corrected RMSE (SC-RMSE) Mean Error (ME), and Standard Deviation Error (SDE). Artificial Intelligence (AI) techniques have experienced great success in the field of path-loss predictions in recent time. Majority of the researches conducted on path-loss predictions using AI techniques have been done at VHF and GSM frequency $(900 \mathrm{MHz})$, very few have been done at UHF band indicating that AI techniques have not been majorly investigated at higher frequency. Based on this assertion and the peculiarity of the environment under consideration, it is therefore necessary to investigate the efficiency of ANFIS for its adaptability in Ibadan, Nigeria at $2100 \mathrm{MHz}$.

\subsection{Determination of path-loss models}

Path-loss models are mathematical expressions used in design and predictions of radio signal attenuation. These models determine path-loss by calculating the difference between the transmitted power and the received power at any distance [8]. These models are categorized into three; deterministic, random and Empirical model $[9,13]$.

\subsubsection{Deterministic model}

These models are based on ray optical laws. They approximate reflection, diffraction and scattering of the signal using simple geometric equations. There must be adequate knowledge of the position of obstacles for this model to be accurate. However, the model has computational complexity which makes it inapplicable as a general tool for path-loss modeling except in indoor environments and rural areas where obstacles are very minimal $[3,13,19]$. 


\subsubsection{Random models}

These models are derived from randomness of the environment due to propagation mechanisms which make the wireless signal unpredictable [6,8]. As a result of this, statistical approach is adopted to predict the signal. The approach is known as random models.

\subsubsection{Empirical models}

Empirical models are based on real time measurement at a certain frequency range and a given distance, these models are limited to the geographical locations where the measurements are taken which makes their accuracy questionable when applied to other environments $[2,11,18]$. Therefore, optimization approach must be carried out for proper adaptation of these models to other environment. The optimized model is the used as a reference for comparison with the measured data

\subsection{Applicability of path-loss models in Ibadan, Nigeria.}

In this session, the applicability of some existing models for path-loss prediction in the environment under consideration is investigated using the base station parameters of the three BTSs $\left(B T S_{1}, B T S_{2}\right.$ and $\left.B T S_{3}\right)$ inIbadan. Details of the transmitting parameters are contained in Table 1.

Table 1. Test Site Parameters

\begin{tabular}{|c|c|c|c|}
\hline Parameters & $B T S_{1}-$ Ojo & $B T S_{2}$ - Challenge & $B T S_{3}-$ Dugbe \\
\hline Operating Frequency & $2100 \mathrm{MHZ}$ & $2100 \mathrm{MHZ}$ & $2100 \mathrm{MHZ}$ \\
\hline Transmitting Antenna Height & $28 \mathrm{~m}$ & $35 \mathrm{~m}$ & $30 \mathrm{~m}$ \\
\hline Receiving Antenna Height & $2 \mathrm{dBi}$ & $2 \mathrm{dBi}$ & $2 \mathrm{dBi}$ \\
\hline Receiving Antenna Height & $1.5 \mathrm{~m}$ & $1.5 \mathrm{~m}$ & $1.5 \mathrm{~m}$ \\
\hline
\end{tabular}

\subsubsection{Applicability Okumura-Hata Model}

Okumura-Hata model developed empirical formulas valid over a frequency range of $150 \mathrm{MHz}-1500 \mathrm{MHz}$ [1,5]. Path-loss ' $P_{\text {Loss }}$ ' model described by Okumura-Hata model for urban environment is given by $[1,16]$ as:

$$
P_{\text {Loss }(\text { oku })}(d B)=69.55+26.16 \log _{10} f_{c}-13.82 \log _{10} h_{t}-a\left(h_{r}\right)+\left(44.9-6.55 \log _{10} h_{t}\right) \log _{10} T R_{D}
$$

where:

$h_{r}$ is the receiving antenna height

$h_{t}$ is the transmitting antenna height

$f_{c}$ is the frequency in $\mathrm{MHz}$,

$T R_{D}$ is the separation between the transmitter and the receiver,

$a\left(h_{r}\right)$ is the correction factor mobile station.

Inserting the site parameters of $B T S_{1}$ into equation (1) gives; 


$$
P_{\text {Loss }(o k u)}(d B)=136.46+35.42 \log _{10} T R_{D}
$$

Where

$$
a\left(h_{r}\right)=3.2\left(\log _{10} 11.75 h_{r}\right)^{2}-4.97 d B \quad f_{c}>300
$$

Path-loss is obtained for $B T S_{2}$ as;

$$
P_{\text {Loss }(o k u)}(d B)=135.12+34.79 \log _{10} T R_{D}
$$

Path-loss is obtained for $\mathrm{BTS}_{3}$ as;

$$
P_{\text {Loss }(o k u)}(d B)=136.04+35.22 \log _{10} T R_{D}
$$

\subsubsection{Applicability of COST-231 Extension to Hata Model}

COST 231 Extension to Hata Model operates over a frequency range of $500 \mathrm{MHz}$ to $2000 \mathrm{MHz}$. Though, there is correction factor for adjustment. The ' $P_{\text {Loss }}$ ' model is given by $[1,5]$ as:

$$
P_{\text {Loss }(\cos t)}(d B)=46.3+33.9 \log _{10} f_{c}-13.82 \log _{10} h_{t}-a\left(h_{r}\right)+\left(44.9-6.55 \log _{10} h_{t}\right) \log _{10}\left(T R_{D}\right)+K_{m}
$$

where:

$h_{r}$ is the receiving antenna height

$h_{t}$ is the transmitting antenna height

$f_{c}$ is the frequency in $\mathrm{MHz}$,

$T R_{D}$ is the separation between the transmitter and the receiver,

$a\left(h_{r}\right)$ is the correction factor mobile station.

$K_{m}$ is the correction factors, and has a value of ' 3 ' for urban areas.

Inserting the site parameters of $B T S_{1}$ in equation (5) gives;

$P_{\text {Loss }(\cos t)}(d B)=141.92+35.42 \log _{10} T R_{D}$

For $B T S_{2}$, path-loss ' $P_{\text {Loss }}$ ' is obtained as;

$P_{\text {Loss }(\cos t)}(d B)=140.58+34.79 \log _{10} T R_{D}$

For $B T S_{3}$, path-loss ' $P_{\text {Loss }}$ ' is obtained as;

$$
P_{\text {Loss }(\text { cost })}(d B)=141.51+35.22 \log _{10} T R_{D}
$$




\subsubsection{Free space models}

Free space propagation assumes there is no obstruction along the path and the signal propagate on a straight line. The ' $P_{\text {Loss }}$ ' for free space propagation is given by $[1,12]$ as:

$$
P_{\text {Loss }(\text { free })}(d B)=32.48+20 \log _{10} T R_{D}+20 \log _{10}(f)
$$

Where $\left(T R_{D}\right)$ is the separation between the transmitter and the receiver in meters $(\mathrm{m})$ and ' $f$ ' is the frequency in MHz.

Inserting the site parameters, path-loss ' $P_{\text {Loss }}$ ' is obtained for $B T S_{1}, B T S_{2}$ and $B T S_{3}$ as;

$$
P_{\text {Loss }(\text { free })}(d B)=98.92+20 \log _{10} T R_{D}
$$

\section{Methodology}

The procedure for data collection involves Equipment Setup inside a vehicle. Information related to the BTS such as gains of the transmitting and receiving antennas, height of antennas and transmitted power are recorded for link budgeting.

\subsection{Equipment Setup}

Three BTSs in Ibadan, Nigeria at (longitude E 3' 53.1060' and latitude N 7'27.2558'), (longitude E 3'50.4361'and latitude N 7' 23.0678') and (longitude E 3' 53.1060' and latitude N 7' 21.258') that have omnidirectional antennas and transmit a continuous wave signal are used for the investigation. The mobile receiver used is a 3G Ericson (W995) TEMS Phone connected to a dual core computer system as shown in Fig. 1. The computer system is integrated with TEMS software capable of mapping Received Signal Strength (RSS) with Global Positioning System (GPS). During the Drive Test campaign, transmitting parameters such as transmitted power, antenna gain, antenna height and the carrier frequency are recorded for every base station. TEMS software is locked on each of the transmitting base station to record RSS and the coordinate of each test point in the direction of the sectors. The receiver is kept at height of $1.5 \mathrm{~m}$. The vehicle is driven away from the base stations at an average speed of $40 \mathrm{~km} / \mathrm{h}$. 10 Measurements samples of RSS are taken for each BTS between $100 \mathrm{~m}$ and $1 \mathrm{~km}$. Seventy percent $(70 \%)$ of the data is used for training and $30 \%$ for testing. Details of the measurement are presented in Table 2.

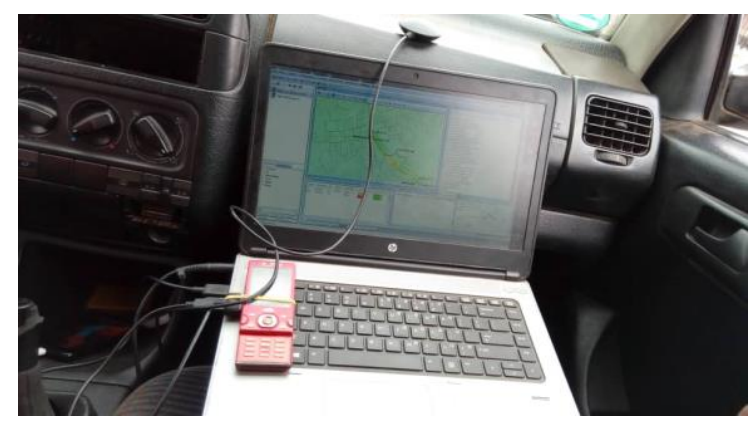

Fig.1. Equipment Set-up 
Table 2. Measured values at different distances for $B T S_{1}-$ Ojo, $B T S_{2}-$ Challenge and $B T S_{3}-$ Dugbe

\begin{tabular}{cccc}
\hline Distance $(\mathrm{m})$ & $B T S_{1}-$ Ojo & $B T S_{2}-$ Challenge & $B T S_{3}-$ Dugbe \\
\hline 100 & 97 & 98 & 96 \\
200 & 105 & 103 & 102 \\
300 & 110 & 105 & 106 \\
400 & 113 & 109 & 110 \\
500 & 117 & 112 & 113 \\
600 & 120 & 116 & 117 \\
700 & 122 & 120 & 121 \\
800 & 124 & 123 & 126 \\
900 & 129 & 126 & 128 \\
1000 & 133 & 129 & 136 \\
\hline
\end{tabular}

\subsection{Development of Adaptive Neuro-Fuzzy Inference System}

ANFIS is the combination of ANN and FL $[4,14,15]$. ANFIS structure has five layers driven by feed forward neural network that utilizes Sugeno 'If-then-rules' [14,15]. Fig. 2 shows the basic ANFIS structure with a single input layer, three hidden layers and one output layer. There are two fuzzy sets used in classifying each of the input. Input ' $x_{1}$ ' has fuzzy sets ' $A_{1}$ ' and ' $A_{2}$ ', input ' $x_{2}$ ' has fuzzy sets ' $B_{1}$ ' and ' $B_{2}$ ', input ' $x_{3}$ ' has fuzzy sets ' $C_{1}$ ' and ' $C_{2}$ and input ' $x_{4}$ ' has fuzzy sets ' $D_{1}$ ' and ' $D_{2}$ '. There are sixteen (16) Sugeno 'If then rule' defined of the form;

$R^{1}$ : If $x_{1}=A_{i,} x_{2}=B_{i,}, x_{3}=C_{i}, x_{4}=D_{i}$, then $Y_{l}=A_{i} x_{1}+B_{i} x_{2}+C_{i} x_{3}+D_{i} x_{4}+E_{i} \quad$ for $l=1, \ldots, 16$

Where $\left(A_{i}, B_{i}, C_{i}, D_{i}, E_{i}\right)$ are called consequent parameters.

\section{Layer 1}

This layer defines the membership grades for each set of input vectors. Usually, it is represented by a Gaussian function. The $i^{t h}$ node of Layer 1 has an output " $O P_{1, i}$ " defined by [15] as:

$$
\begin{aligned}
& \text { For input } x_{1} ; O P_{1, i}=\mu_{A i}(x)=\exp \left(-\left(\frac{x_{1}-c_{i}}{\sigma_{i}}\right)^{2}\right) \quad \text { for } \mathrm{i}=1,2 \\
& \text { For input } x_{2} ; O P_{1, i}=\mu_{B i}(x)=\exp \left(-\left(\frac{x_{2}-c_{i}}{\sigma_{i}}\right)^{2}\right) \quad \text { for } \mathrm{i}=1,2
\end{aligned}
$$

where :

$\left(\sigma_{i}, c_{i}\right)$ are called premise parameters that define the shape of the membership functions,

$\mu_{A i}, \mu_{B i}, \mu_{C i}$ and $\mu_{D i}$ are the input membership grades defined,

$c_{i}$ determines the center of the corresponding membership function. 


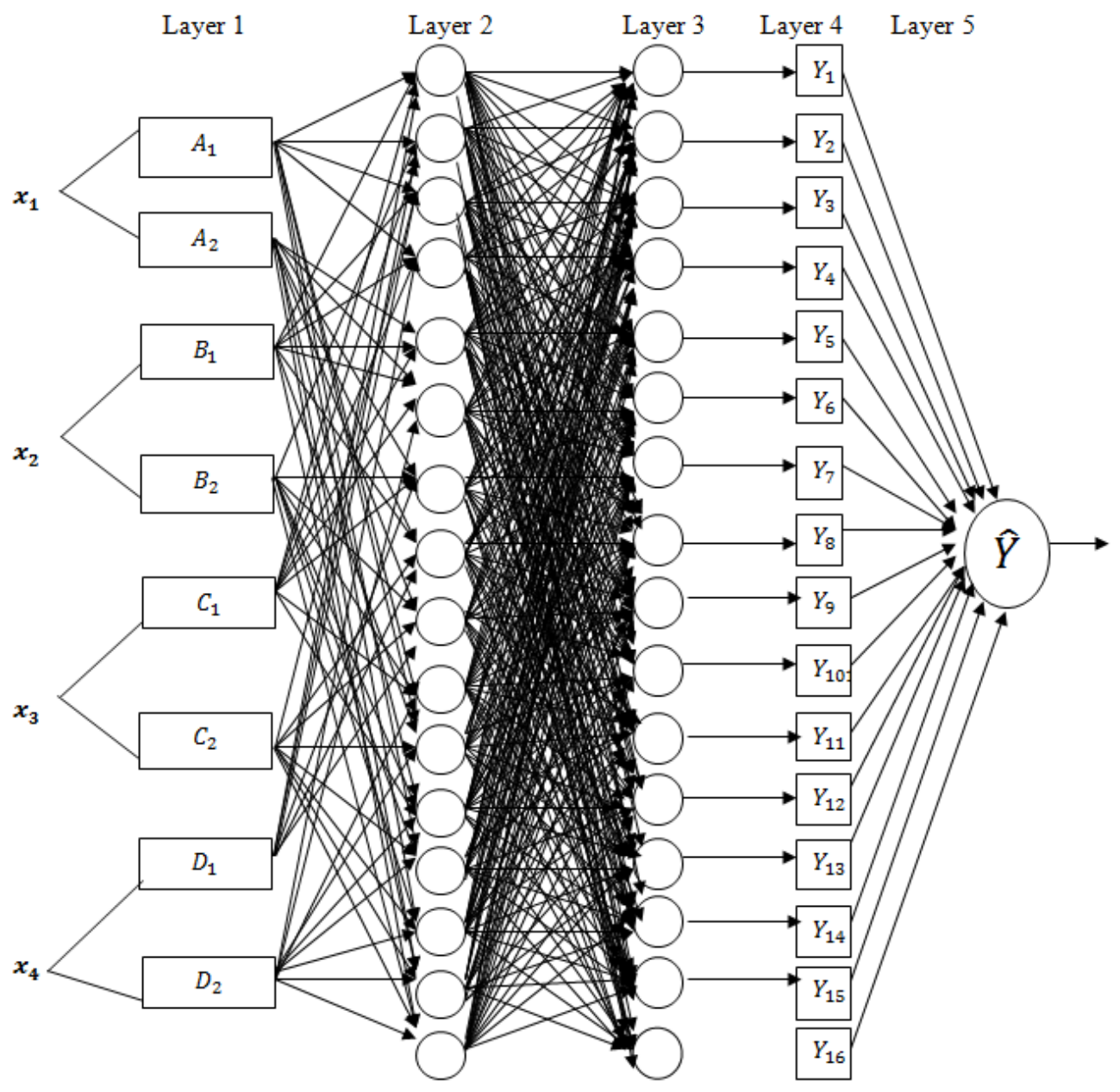

Fig. 2. ANFIS Structure

\section{Layer 2 (Product layer)}

Takes the product of the incoming membership grades with the output " $O P_{2, i}$ " of the $i^{\text {th }}$ node given by [15] as:

$$
O P_{2, i}=w_{l}=\mu_{A i}\left(x_{1}\right) * \mu_{B i}\left(x_{2}\right) * \mu_{C i}\left(x_{3}\right) * \mu_{D i}\left(x_{4}\right) \quad \text { for } l=1, \ldots, 16
$$

\section{Layer 3 (Normalized layer)}

The layer determines the ratio of the ith rule's firing strength to the sum of all rules' firing strength. The output ' $O P_{3, i}$ ' is defined as:

$$
O P_{3, i}=\overline{w_{l}}=\frac{w_{l}}{\sum_{l=1}^{n=16} w_{l}} \quad \text { for } l=1 \ldots 16
$$


where; $\overline{w_{l}}$ is the normalized firing strength,

$w_{l}$ is the firing strength of the $i^{\text {th }}$ rule,

\section{Layer 4}

The layer computes the contribution of each rule to the overall output and provides output values resulting from the inference of the rules. The output ' $O P_{4, i}$ ' of the node is given as:

$$
O P_{4, i}=\bar{w}_{l} Y_{l}=\bar{w}_{l}\left(A_{i} x_{1}+B_{i} x_{2}+C_{i} x_{3}+D_{i} x_{4}+E_{i}\right) \quad \text { for } l=1, \ldots, 16
$$

where:

$\left(A_{i}, B_{i}, C_{i}, D_{i}, E_{i}\right)$ are called consequent parameters, $x_{1}, x_{2}, x_{1}$ and $x_{2}$ are the input variables,

$\overline{w_{l}}$ is the output of layer 3 .

\section{Layer 5 (Final Output)}

This layer computes the overall output as a summation of all incoming signal. The output of the ith node of this layer ' $O P_{5, i}$ ' is given as:

$$
O P_{5, i}=\sum_{l=1}^{16} \overline{w_{l}} Y_{l}
$$

Where $Y_{l}$ is the final part of the inference rule governing the path-loss defined as;

$$
Y_{l}=\left(A_{i} x_{1}+B_{i} x_{2}+C_{i} x_{3}+D_{i} x_{4}+E_{i}\right)
$$

In Training ANFIS for the path loss model development, the input variables are height of the transmitting antenna, height of the receiving antenna, frequency and distance represented as $\left(x_{1}, x_{2}, x_{3}, x_{4}\right)$. Hybrid learning algorithm that combines LSE and GD method is used for the training. LSE are used to update the sets of consequent parameters while GD updates the premise parameters [14,15]. The training continues till error between the predicted and the measured values are minimized based on the relation. The performance of the developed model is compared to Okumura-Hata, COST-231 and free space model.

\section{Results and discussion}

Figs. 3. to 5. present path-loss versus distance for ANFIS model, Okumura-Hata model, COST-231 model, free space model and measured values for $B T S_{1}(\mathrm{Ojo}), B T S_{2}$ (Challenge) and $B T S_{3}$ (Dugbe). Fig. 3. presents path-loss against distance for ANFIS, measured, Okumura-Hata, COST 231 and free space models for $B T S_{1}$. At $500 \mathrm{~m}$ from the base station, path-loss values obtained are 117,114, 122, 127 and $91 \mathrm{~dB}$ for ANFIS, measured, Okumura-Hata, COST-231 and free space. Fig. 4 depicts path-loss values against distance for ANFIS, measured, Okumura-Hata, COST-231 and free space models for $B T S_{2}$. At $500 \mathrm{~m}$ from the base station, path-loss values obtained are 115, 112, 126, 131 and $93 \mathrm{~dB}$ for ANFIS, measured, Okumura-Hata, COST 231 and free space models, respectively. Fig. 5 shows path-loss values against distance for ANFIS, measured, Okumura-Hata, COST 231 and free space models for $B T S_{3}$. At $500 \mathrm{~m}$ from the base station, path-loss values obtained are 117, 113, 125, 131 and $93 \mathrm{~dB}$ for ANFIS, measured, Okumura-Hata, COST 231 and free space models, respectively. 


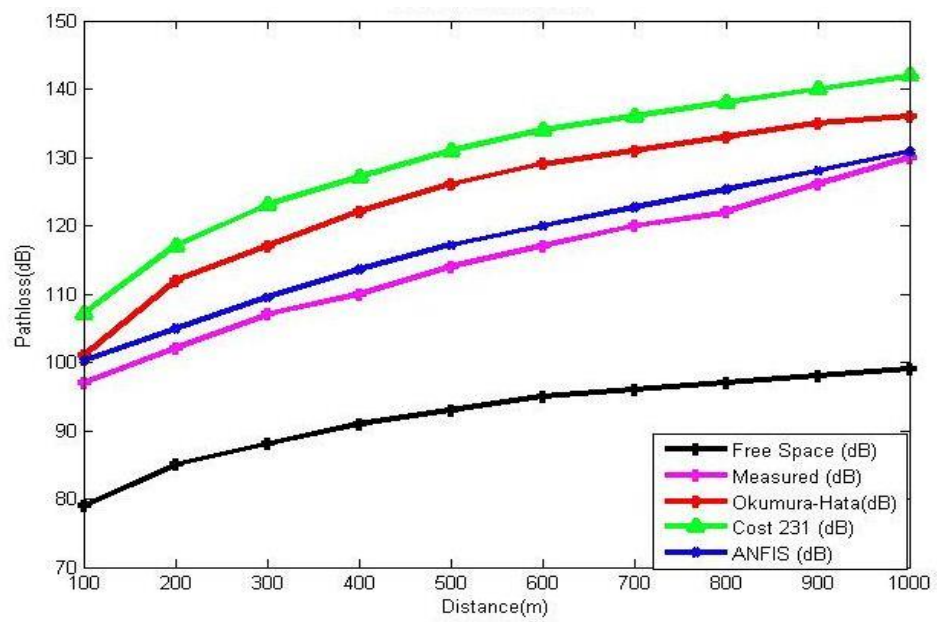

Fig. 3. Path-loss versus distance for ANFIS, Measured, COST 231, Okumura-Hata and Free space models for BTS 1 (Ojo)

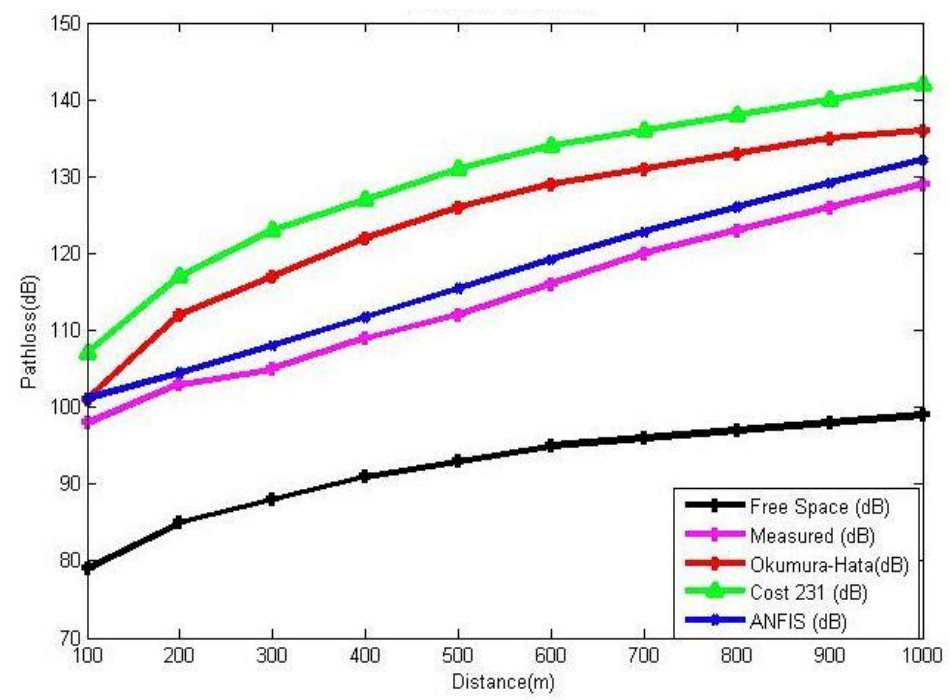

Fig. 4. Path-loss versus distance for ANFIS, measured, COST-231, Okumura-Hata and Free space models for BTS $_{2}($ Challenge) 


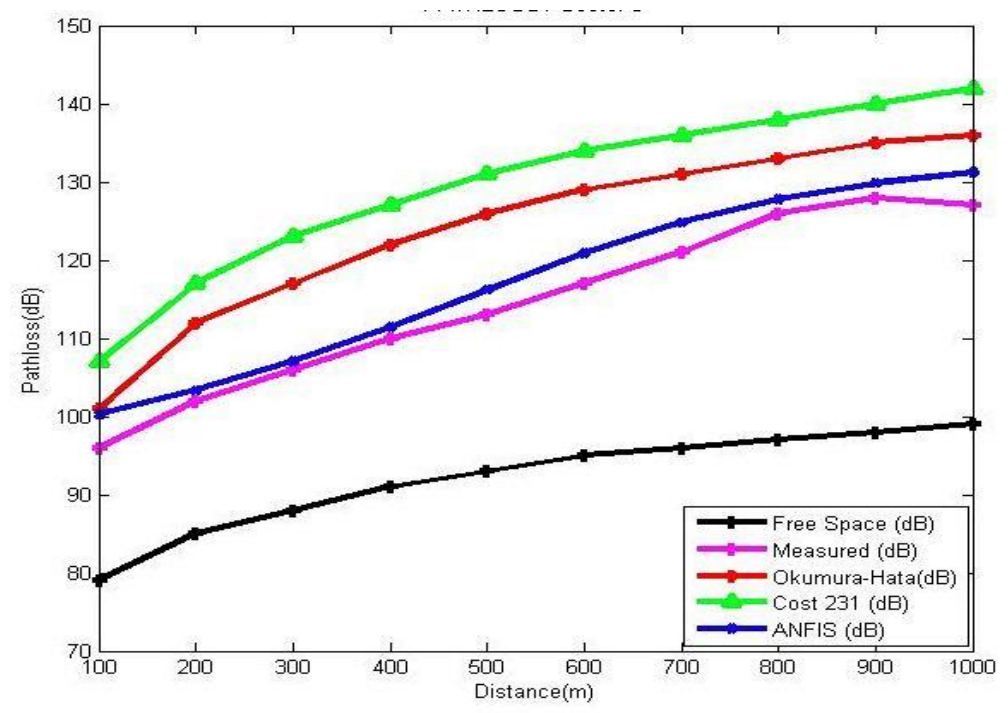

Fig. 5. Path-loss versus distance for ANFIS, measured, COST-231, Okumura-Hata and Free space models for BTS 3 (Dugbe)

The Average MSE (AMSE) obtained at BTS 1 are 106.16, 232.03, 500.13 and 7.99 for Okumura-Hata, COST-231, free space and ANFIS models, respectively. For $B T S_{2}$, AMSE values are 124.96, 261.30, 515.53 and 8.71 for Okumura-Hata, COST-231, free space and ANFIS models, respectively while the corresponding values for $B T S_{3}$ are $131.80,199.10,521.47$ and 8.74 , respectively. The average RMSE (ARMSE) values obtained from $B T S_{1}$ are 10.30, 15.23, 22.36 and 2.82 for Okumura-Hata, COST-231, free space and ANFIS models, respectively while ARMSE values of 11.18, 16.17, 22.70 and 2.95 are obtained from $B T S_{2}$ for Okumura-Hata, COST 231, free space and ANFIS models, respectively. The corresponding values of 11.48, 14.11, 22.84 and 2.96 are obtained from $B T S_{3}$. The results are also contained in Tables 3 and 4 for $B T S_{1}$ to $B T S_{3}$. ANFIS models give the lowest AMSE and ARMSE indicating the best model to predict the path loss in these environments. This is due to reduction in error by ANFIS.

Table 3. Average MSE results for the three BTS

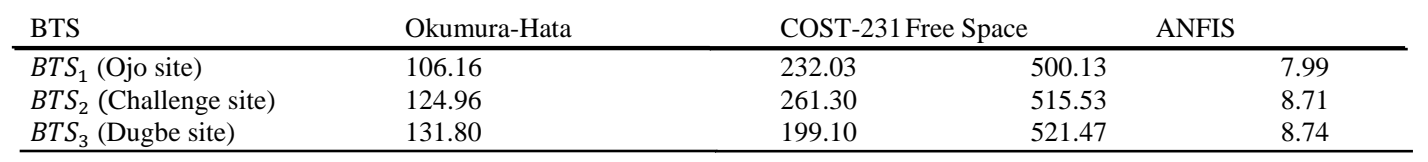

Table 4. Average RMSE results for the three BTS

\begin{tabular}{llccl}
\hline BTS & Okumura-Hata & & COST-231Free Space & ANFIS \\
\hline$B T S_{1}$ (Ojo site) & 10.30 & 15.23 & 22.36 & 2.82 \\
$B T S_{2}$ (Challenge site) & 11.18 & 16.17 & 22.70 & 2.95 \\
$B T S_{3}$ (Dugbe site) & 11.48 & 14.11 & 22.84 & 2.96 \\
\hline
\end{tabular}




\section{Conclusions}

Adaptive Neuro-Fuzzy Inference Systems approach has been used to develop a path-loss model for HSPA network signal in Ibadan, Nigeria. RSS data are measured from three selected BTSed from three selected BTSs located at Ojo, Challenge and Dugbe areas of Ibadan using drive test. A five layer ANFIS structure is developed and trained using LSE and GD algorithm. HSPA base station parameters such as antennas height, carrier frequency, and separation of the transmitting and receiving antennas are used as input variables to train ANFIS. The development platform is MATLAB R2012a version on 64 bits' dual core computer system. The base station parameters are used to investigate the suitability of Okumura-Hata, COST-231 and free space model. The performance of the developed model is evaluated using RMSE and MSE and compared with Okumura-Hata, COST-231 and free space model. The results show that, errors related to ANFIS model is greatly reduced when compared with the existing models.

\section{References}

[1] Goldsmith A, Wireless communications, 1sted. Stamford University, California; 2004

[2] Omae M O, Ndungu, E N, and Kibet P L, The Application of ANFIS-PSO trained in Signal Propagation Modeling for Indoor Wireless Communication Networks, A Review, International Journal of Scientific \& Engineering Research, 2014; 5(2):180-191

[3] Okorogu V N, Onyishi D U, Nwalozie G C, Utebor N N, Empirical Characterization of Propagation Path Loss and Performance Evaluation for Co-Site Urban Environment, International Journal of Computer Applications, 2013;70(10):34-41.

[4] Hazlina H, An Exploration of the Adaptive Neuro-Fuzzy Inference System (ANFIS) in Modelling Survival, P.hd Thesis, School of Computer Science, University of Nottingham, 2013

[5] Rappaport T, Wireless Communications, Principles and Practices, $2^{\text {nd }}$ ed. Prentice Hall PTR, Upper Saddle River, New Jersey, 2002.

[6] Raturi P, Gupta V, and Eram S, Proposed Propagation Model for Dehradun Region, International Journal of soft Computing and Engineering (IJSCE), 2014; 3 (6):236-240

[7] Ramesh V, Thangaraj T S, and Prasad J V, An Efficient Path Loss Prediction mechanism in Wireless Communication Network Using Fuzzy Logic, International Journal of Advanced Research in Computer Science and Software Engineering, 2012; 2(1): 289-298

[8] Rakesh, N., Srivasta, S. K., "A study of path loss analysis for GSM Mobile Network for Urban, Rural AndSuburban Regions of Kernataka State", International journal of Distributed and parallel system (IJDPS) 2013; 4 (1):56-61

[9] Abhayawardhana V S, Wassell I J, Crosby D, Sellars M P, Brown M G, Comparison of Empirical Propagation Path Loss Models for Fixed Wireless Access Systems, International Journal of Scientific \& Engineering Research, 2009; 4 (2):270-275

[10] Syed M A, Muhammed M, Applicability of Path Loss Prediction in Macrocellular Environment, 2015; 4 (9):31-34

[11] Omae, M O, Ndungu E N, and Kibet P L, Tarus H, Artificial Intelligence Approach to Signal Propagation Modeling for Outdoor to Indoor Wireless Communication Networks, A Proposed Study International Journal of Scientific \& Engineering Research, 2012; 4 (4): 289-298.

[12] Megha, D., Rohit, S., Determination of Path-loss using RF Link Budget in Suburban Macrocell Environment for GSM Communication System. International Journal of Science and Research (IJSR) 2014; 3 (9):1409-1412

[13] Dominic S., Musa A., Tonga A. "Path Loss Propagation Model Prediction for GSM Mobile Network Planning in Kaduna town", International Journal of Engineering Sciences and Research Technology, (2015); 4 (4): 345-352 
[14] Wang Z, Palade V. \& Xu Y, Neuro-Fuzzy ensemble approach for microarray cancer gene expression data analysis, In Evolving Fuzzy Systems, International Symposium on, 2006; 36(1): 2-17

[15] Jang, J R, Adaptive Network -Based Fuzzy Inference Systems. IEEE Transactions on Systems, Man and Cybernetics, 1993; 23 (4): 31-37.

[16] Ujjawal, B., and Bhagirathi, P. A Review On Path Loss Models for Suburban Regions Using Fuzzy Logic, International Journal of Computer Engineering \& Science, 2014: 4 (2): 74-78

[17] Nazmat T. S, Nasir, F., Segun I. P, Muhammed A.S, Abdulkarim A. O, Lukman A.O, Carlos T.C. Path loss predictions for multi-transmitter radio propagation in VHF bands using Adaptive Neuro-Fuzzy

[18] Adeyemo, Z.K., Ogunremi, O.K and Akande, A.O., Genetic Algorithm based pathloss optimization for Long Term Evolution in Lagos, Nigeria, international journal of applied science and technology, 2016, 6(2): 70-88.

[19] Adeyemo, Z.K., Ogunremi, O.K and Ojedokun, I.A. Optimization of Okumura-Hata model for long term evolution network deployment in Lagos, Nigeria, International Journal on Communication Antenna and Propagation, 2016, 6(3): 146-152.

\section{Authors' Profiles}

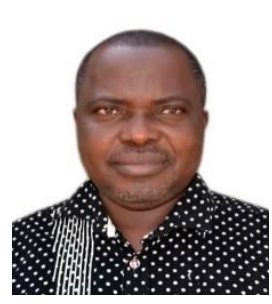

Zachaeus K. Adeyemo obtained the B.Eng. and M.Eng. degrees in Electrical Engineering from University of Ilorin, Ilorin Nigeria and his Ph.D. degree in Electronic and Electrical Engineering in 2009 from Ladoke Akintola University of Technology (LAUTECH), Ogbomoso, Nigeria. Prof. Adeyemo is a Professor of Communication Engineering at the Department of Electronic and Electrical Engineering, LAUTECH, Ogbomoso. He is a member of the IEEE and registered member of Council for the Regulation of Engineering in Nigeria (COREN). His research interest is on signal processing in wireless communications.

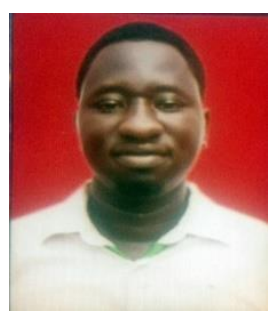

Tolulope O Olawuyi received his B.Tech degree in Electronic and Electrical Engineering in 2009 from Ladoke Akintola University of Technology (LAUTECH), Ogbomoso, Nigeria. He is currently pursuing his M.Tech in wireless communication from the same University. His research interest is signal processing in mobile communication

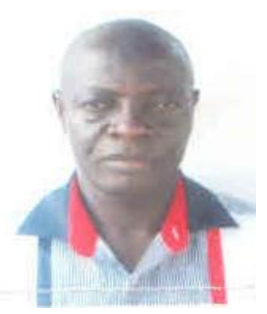

Olasunkanmi F. Oseni received the B.Eng. and M.Eng. degrees in Electrical Engineering from University of Ilorin, Ilorin Nigeria and his Ph.D. degree in Electronic and Electrical Engineering in 2018 from Ladoke Akintola University of Technology (LAUTECH), Ogbomoso, Nigeria. He is a member of the IEEE and registered member of Council for the Regulation of Engineering in Nigeria (COREN). 


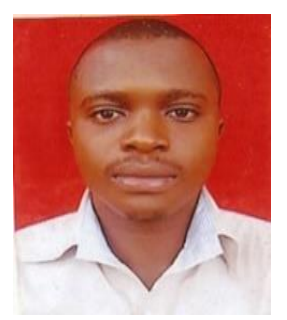

Samson I. Ojo received his B.Tech and M.Tech degrees in Electronic and Electrical Engineering in 2011 and 2018, respectively, from Ladoke Akintola University of Technology (LAUTECH), Ogbomoso, Nigeria. He is a registered member of Council for the Regulation of Engineering in Nigeria (COREN). Presently, is a Ph.D student at the Department of Electronic and Electrical Engineering, LAUTECH. His research interest is signal processing in mobile communication.

How to cite this paper: Adeyemo Z.K, Olawuyi T.O, Oseni O.F, Ojo S.I, " Development of a Path-loss Prediction Model Using Adaptive Neuro-fuzzy Inference System", International Journal of Wireless and Microwave Technologies(IJWMT), Vol.9, No.6, pp. 40-53, 2019.DOI: 10.5815/ijwmt.2019.06.05 